\title{
Una nuova specie del genere Halbherria Conci \& Tamanini, 1951 del massiccio del Monte Baldo e considerazioni sulla "barriera" biogeografica della Valle dell'Adige (Coleoptera Cholevidae Leptodirinae)
}

\begin{abstract}
Riassunto: Nel presente lavoro viene descritta Halbherria carlini nuova specie del massiccio del Monte Baldo, appartenente ad un genere di Cholevidae Leptodirinae finora noto del settore delle Prealpi Venete compreso tra i fiumi Brenta e Adige. La scoperta di Halbherria sul M. Baldo riapre la discussione sul ruolo di "barriera" della Valle dell'Adige, che potrebbe essere stata superata da parte dei precursori di alcuni taxa alla fine del Pliocene, comunque anteriormente alle glaciazioni pleistoceniche, quando l'assetto idrografico della valle era diverso dall'attuale.

Abstract: A new species of the genus Halbherria Conci \& Tamanini, 1951 from the Monte Baldo massif and remarks on the biogeographic "barrier" of the Adige Valley (Coleoptera Cholevidae Leptodirinae).

Halbherria carlini new species is described from the Monte Baldo massif. The new species belongs to a genus of Cholevidae Leptodirinae so far known from the Venetian pre-Alps, between the rivers Brenta and Adige. The discovery of Halbherria on Monte Baldo reopens the discussion about the "barrier" role of the Adige Valley, which may have been exceeded by the ancestors of some taxa at the end of the Pliocene, or in any case before the Pleistocene glaciations, when the hydrographic asset of the Valley was different from present.
\end{abstract}

Key words: Cholevidae, Leptodirinae, new species, taxonomy, biogeography, Adige Valley.

\section{INTRODUZIONE}

Al genere Halbherria Conci \& Tamanini, 1951 appartengono, secondo le attuali conoscenze, nove specie che, sulla base della morfologia dell'edeago, si possono suddividere fra tre distinti gruppi (Giachino \& Vailati, 2005): un "gruppo di $H$. stefani”, comprendente $H$. stefani (Breit, 1914), $H$. zorzii (Ruffo, 1950) e H. genesti Giachino \& Vailati, 2005; un "gruppo di H. tamaninii", comprendente $H$. tamaninii (J. Müller, 1931), H. pacei Piva, 1988, H. pivai Giachino \& Vailati, 2005, H. pasubiana Giachino \& Vailati, 2005 e H. vericoi Piva, 1984; infine, un "gruppo di H. cimbrica", monospecifico, comprendente solo $H$. cimbrica Piva, 1985, la più orientale e alquanto isolata per la morfologia dell'edeago, dalle altre specie.

L'intero genere risulta distribuito, sempre in base alle attuali conoscenze, nelle Prealpi Venete Occidentali, nel territorio compreso tra le valli dei fiumi Brenta e Adige; in particolare, tutte le nove specie note ad oggi sono distribuite sui rilievi dell'altopiano dei Sette Comuni, di Folgaria, del Pasubio, del Carega, fino ad arrivare agli alti Lessini. Il nuovo taxon qui descritto, scoperto grazie alle ricerche condotte da Andrea Carlin di Pergine Valsugana, riveste un partico- lare interesse, essendo la prima specie di Halbherria presente sul Monte Baldo, quindi a occidente della valle del Fiume Adige. Le implicazioni biogeografiche di questa presenza vengono discusse nelle considerazioni finali di questa nota. È questo l'ennesimo caso che si aggiunge ad altri che in anni abbastanza recenti ci hanno ormai abituati a non sorprenderci più di tanto di fronte alle continue novità. Il grande massiccio del Monte Baldo, per quanto intensamente esplorato da molti decenni da parte di innumerevoli naturalisti, ci ricorda ancora una volta di quanto siano limitate le nostre conoscenze, anche là dove spesso crediamo, presuntuosamente, di sapere quasi tutto.

\section{MATERIALI E METODI}

La descrizione della nuova specie è basata su esemplari provenienti dalle raccolte effettuate da Andrea Carlin in una caverna militare nei dintorni della cima del M. Altissimo di Nago.

Le misure e i disegni sono stati eseguiti utilizzando micrometri oculari su stereomicroscopio Wild M3 e camera lucida su microscopio Leitz Dialux. I preparati, realizzati previa deidratazione e diafanizzazione, sono montati in balsamo del Canada su cartellini trasparenti uniti allo spillo dei relativi esemplari.

*Dante Vailati, Via Interna 8, 25127 Brescia, Italia. E-mail: dante.vailati@libero.it 
seguenti:

Le abbreviazioni usate nel testo sono le

HT: Holotypus;

PT, PTT: Paratypus, Paratypi;

TL: lunghezza totale del corpo, misurata a capo reclinato;

AL: $\quad$ lunghezza dell'antenna;

PL: $\quad$ lunghezza del pronoto, misurata lungo la linea mediana;

PW: massima larghezza del pronoto;

EL: $\quad$ lunghezza delle elitre;

EW: larghezza massima delle elitre considerate insieme;

TL/AL: rapporto lunghezza del corpo/lunghezza dell'antenna;

PW/PL: rapporto massima larghezza del pronoto/ lunghezza del pronoto;

EL/EW: rapporto lunghezza delle elitre/lunghezza delle elitre.

CCa: Collezione A. Carlin (Pergine Valsugana, TN)

CGi: $\quad$ Collezione P.M. Giachino (Torino)

CVa: Collezione D. Vailati (Brescia)

\section{Halbherria carlini n. sp.}

LocAlitÀ TIPICA. Trentino, Brentonico, M. Altissimo di Nago, cavernetta militare presso il Rifugio D. Chiesa, 1950 m s.1.m.

SERIE TIPICA. Holotypus $\overbrace{}^{\Uparrow}$ : IT, Trentino, Brentonico, M. Altissimo di Nago, cavernetta militare presso il Rifugio D. Chiesa, 1950 m, 21.VIII.2013, A. Carlin legit (CVa). Paratypi: stessa località dell'holotypus, 4qq, 21.VIII.2013, A. Carlin legit (CCa, CGi, CVa); 2 우, 29.VI.2017, A. Carlin legit (CCa); Trentino, M. Altissimo di Nago, cavernetta militare presso il Rifugio D. Chiesa, 1860 m, 1ð̂, 5.V.2017, A. Carlin legit (CCa); 1§̂, 20.VIII.2017, A. Carlin legit (CCa); 2qq, 13.X.2017, A. Carlin legit (CCa).

DiAGNOSI. Un Leptodirino appartenente, per l'insieme dei caratteri, al genere Halbherria e in particolare al "gruppo di H. tamaninii" (sensu Giachino \& Vailati, 2005), per il lobo mediano dell'edeago, in visione laterale, rettilineo, non piegato e non strozzato ventralmente nel terzo basale; in visione dorsale con il quarto distale ristretto a "collo di bottiglia", con i dentini preapicali piccoli e peculiarmente rivolti verso la base, con parameri ben più brevi del lobo mediano e le bacchette sclerificate dell'endofallo grandi, allungate e appuntite distalmente. Si distingue facilmente da tutte le specie note del gruppo soprattutto per i dentini preapicali dell'edeago piccoli, in posizione alquanto arretrata e rivolti verso la base come le alette di una freccia, anziché verso l'esterno o in avanti (Figg. 11-16).

DESCRIZIONE. TL a capo reclinato: HT $\lesssim 3,12 \mathrm{~mm}$, PTT 우 2,97-3,24 mm. Colore testaceo, con zampe, antenne e palpi più chiari. Corpo (Fig. 1) ovalare molto allungato, convesso, poco attenuato all'apice. Antenne (Fig. 2) lunghe, quasi raggiungenti, distese all'indietro, la metà delle elitre (rapporto TL/AL: HT ऽ. 1,62; PTT 우 1,76-1,83).

Lunghezza degli antennomeri in $\mathrm{mm}$ :

त 0,$167 ; 0,170 ; 0,145 ; 0,156 ; 0,167 ; 0,136 ; 0,211$; 0,$117 ; 0,203 ; 0,175 ; 0,245$

ㅇ 0,$156 ; 0,178 ; 0,142 ; 0,145 ; 0,145 ; 0,117 ; 0,213$; 0,$119 ; 0,167 ; 0,178 ; 0,227$

Pronoto trasverso (rapporto PW/PL: HT $ぇ$ 1,52; PTT 우 1,56-1,63), con massima larghezza appena avanti alla base; lati lungamente arrotondati anteriormente, poco o nulla ristretti e non sinuati posteriormente; base bisinuata con angoli arrotondati, poco sporgenti posteriormente.

Elitre allungate e poco attenuate posteriormente (rapporto EL/EW: HT §ै 1,50; PTT 우 1,44-1,49), con massima larghezza circa alla metà, separatamente e regolarmente arrotondate distalmente; stria suturale assente, microscultura rasposa, pubescenza vagamente allineata trasversalmente, soprattutto nel terzo basale, ma in assenza di vere striole trasversali.

Carena mesosternale come in Fig. 3, alta, a profilo anteriore poco curvo, non sfuggente e angolo anteriore quasi retto; profilo inferiore debolmente incavato e seghettato; apofisi posteriore libera, poco allungata e ricoprente solo parzialmente il metasterno.

Protarsi tetrameri nei due sessi, non dilatati nel ふ̂.

Edeago grande, allungato, della forma caratteristica del "gruppo di $H$. tamaninii", con il lobo mediano, in visione laterale (Fig. 5), non strozzato e non flesso ventralmente nel terzo basale, dorsalmente regolarmente curvo per tutta la sua estensione, con apice snello e bruscamente flesso, quasi ad uncino, ventralmente; in visione dorsale (Fig. 4), con i lati lungamente e progressivamente convergenti dalla base circa al quarto distale, poi sinuati e ristretti distalmente a "collo di bottiglia", a lati paralleli e troncato apicalmente. 
Alla base della porzione apicale, tra il settimo e l'ottavo distale del lobo mediano, sono presenti due espansioni laterali conformate come le "alette" di una freccia, di dimensioni alquanto ridotte, ma, a differenza delle altre specie del "gruppo", in cui i dentini sono sporgenti in fuori o leggermente in avanti, tali alette sono rivolte verso la base; inoltre, la loro posizione è ben più arretrata rispetto a quella dei dentini delle altre specie (Figg. 11-16). Apice semplice, non trilobato, troncato e solo leggermente incavato. Lama basale del lobo mediano grande, larga ma relativamente breve. Parameri relativamente gracili, più brevi del lobo mediano, muniti di tre setole, tutte in posizione apicale (Figg. 6 e 7). Sacco interno con armatura rappresentata da due grandi bacchette mediane, lunghe circa quanto i $2 / 3$ del lobo mediano e larghe, curve, falciformi, la cui posizione in rapporto al lobo mediano non è rilevabile in quanto, nell'unico maschio a disposizione, sono estroflesse insieme a tutto il sacco interno (Figg. 4 e 5).
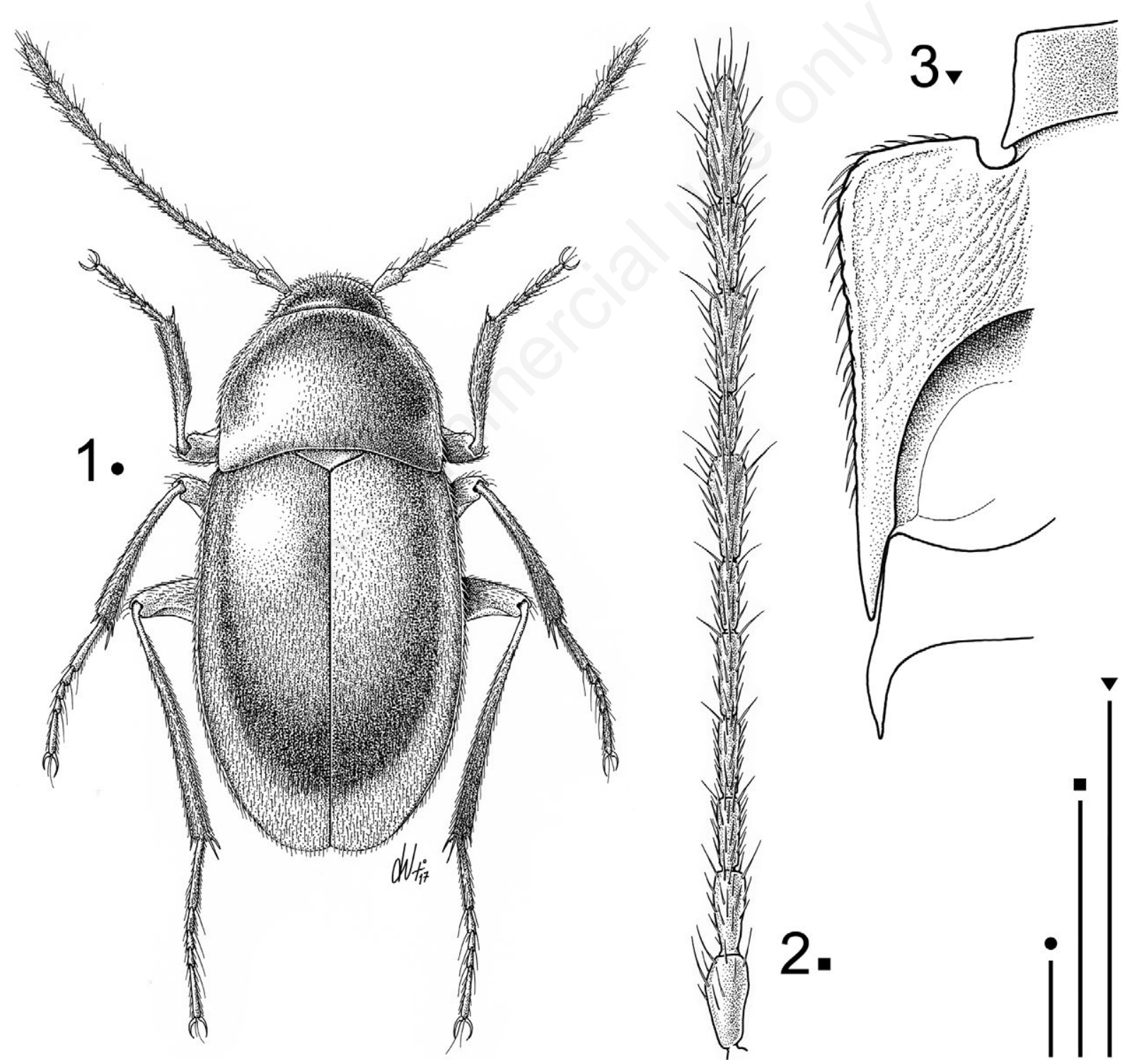

Figg. 1-3. Halbherria carlini n. sp., holotypus $\hat{\partial}$. 1) habitus; 2) antenna; 3) carena mesosternale (scale: 0,5 mm). 

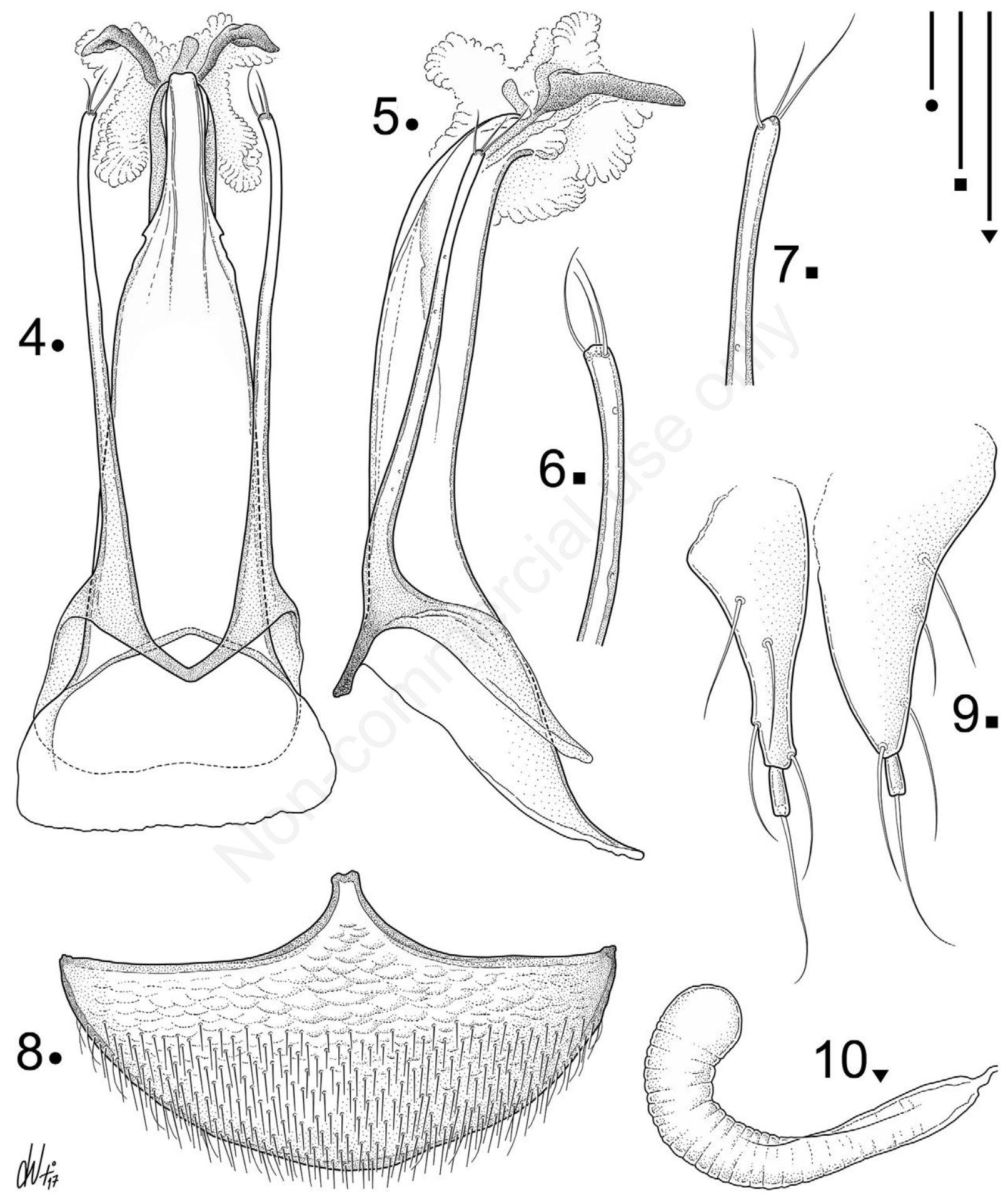

Figg. 4-10. Halbherria carlini n. sp., holotypus $\widehat{\partial}$. 4) edeago in visione dorsale, con endofallo estroflesso; 5) id. in visione laterale; 6) apice del paramero sinistro in visione dorsale; 7) id. in visione laterale; 8) ottavo ventrite della 9 (paratypus); 9) gonostilo; 10) spermateca (scale: $0,1 \mathrm{~mm}$ ). 

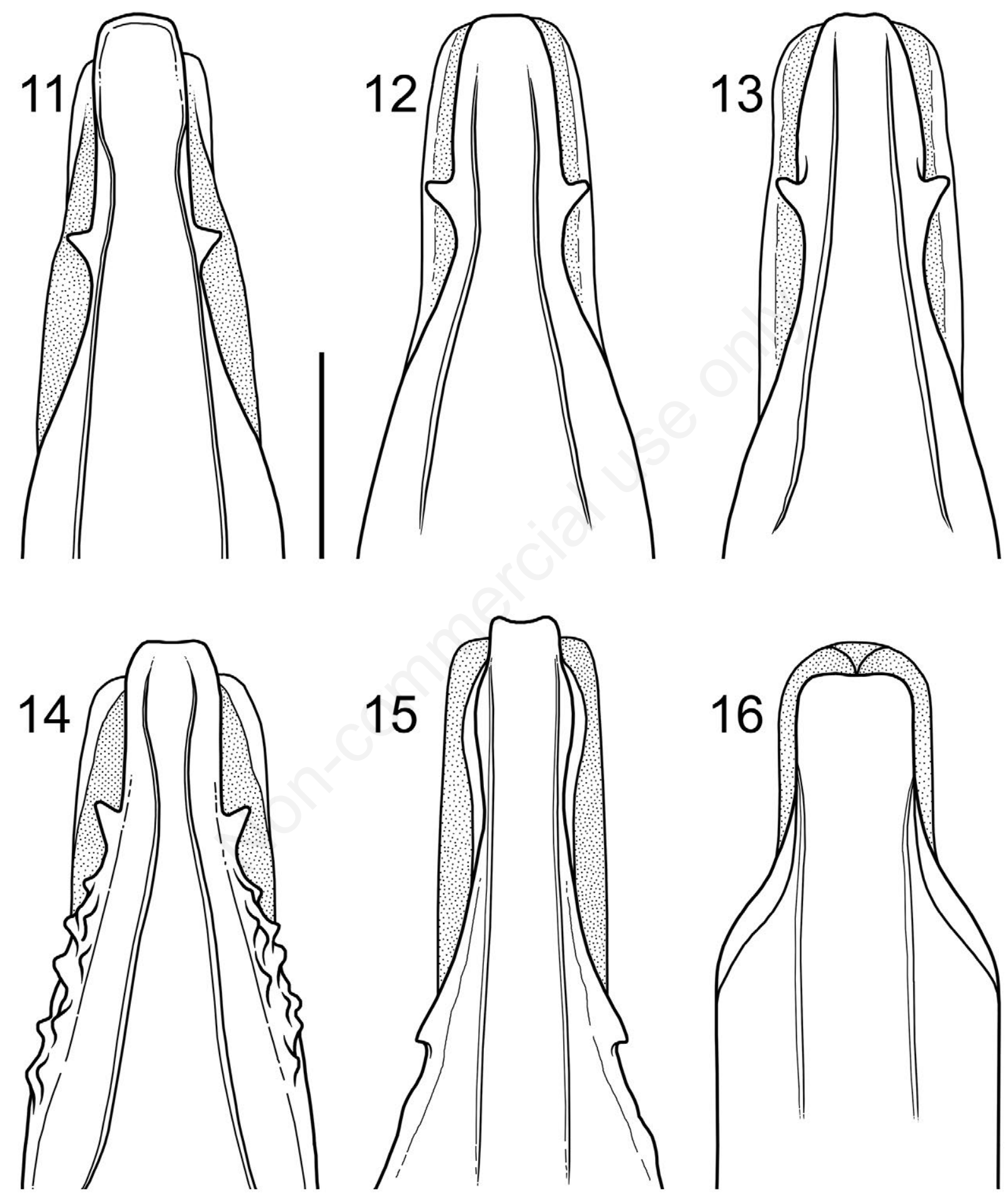

Figg. 11-16. Porzione distale del lobo mediano dell'edeago, in visione dorsale, in Halbherria spp. del "gruppo di H. tamaninii". 11) H. tamaninii; 12) H. pacei; 13) H. pasubiana; 14) H. pivai; 15) H. carlini n. sp.; 16) H. vericoi (ridis. da Piva, 1984) (scala: 0,1 mm). 
Ottavo ventrite nella femmina munito al bordo anteriore di spiculum tozzo, poco sviluppato in lunghezza e con l'apice lievemente bilobato (Fig. 8).

Stilomeri della femmina come in Fig. 9, muniti di una setola apicale relativamente lunga e di due subapicali più brevi.

Spermateca come in Fig. 10, allungata, curva a forma di $\mathrm{C}$, completamente ialina e con porzione distale non particolarmente dilatata.

Etimologia. Dedico con piacere questa nuova specie al suo scopritore, Andrea Carlin di Pèrgine Valsugana (Trento), appassionato e instancabile indagatore della fauna sotterranea delle Venezie.

OSSERVAZIONI. La nuova specie, come evidenziato già nella diagnosi, è ben differenziata dalle congeneri, anche all'interno del "gruppo di $H$. tamananii" cui appartiene; la sua morfologia non richiede altri commenti ai caratteri che non siano desumibili dalla descrizione fornita. Tuttavia è interessante ritornare sulle peculiarità dell'edeago, con le "alette" laterali della porzione distale del lobo mediano rivolte verso la base anziché dei veri e propri "dentini" rivolti in fuori o verso l'apice; non soltanto perché costituisce un carattere che, da solo, rende questo taxon inconfondibile, ma anche perché tale singolarità ci richiama in qualche modo quanto osservato da Piva (1984) all'atto della descrizione di Halbherria vericoi. In quella sede, l'Autore sottolinea giustamente che, nella specie che va descrivendo, sono assolutamente assenti i dentini laterali preapicali dell'edeago, tanto che, ammettendo che $H$. vericoi appartiene inequivocabilmente, per l'insieme dei caratteri, al genere Halbherria, è costretto a variare la chiave dicotomica originale del genere (Conci \& Tamanini, 1951) per potervela inserire. Nel contempo l'Autore rimarca, sia nella descrizione, sia nelle considerazioni conclusive, la presenza di una "profonda piega obliqua sormontata da una evidente espansione laminare". Credo che tale "espansione laminare", si possa interpretare come omologa alle "alette" presenti nella nuova specie, in cui la lamina, anziché essere assolutamente liscia e inerme, si rialza leggermente in due piccoli rilievi angolosi, la stessa lamina che in altre specie si espande evidentemente in modo diverso, fino a portare ai "dentini" rivolti in fuori (come in $H$. tamaninii), o leggermente in avanti (come in H. pacei e in H. pasubiana) o ancora a svilupparsi verso la base, formando la serie di pliche mul- tidenticolate che troviamo in $H$. pivai (Figg. 11-16). L'esasperazione dei "dentini" preapicali, presenti invece nel "gruppo di H. stefani", più robusti, non laminari e decisamente rivolti verso l'apice, è probabilmente legata alla diversa morfologia generale del lobo mediano. Maggiormente enigmatica rimane la morfologia dell'edeago, ad apice trilobato, di Halbherria cimbrica Piva, 1985, più difficilmente avvicinabile a quella delle altre specie.

DistribuZIONe e habitat. Halbherria carlini n. sp. è nota al momento attuale di due sole stazioni fra loro molto prossime, situate non lontano dalla cima del M. Altissimo di Nago, la più settentrionale delle elevazioni della lunga dorsale che costituisce il massiccio del $\mathrm{M}$. Baldo, in territorio trentino. Come H. pasubiana Giachino \& Vailati, 2005 dello stesso "gruppo" e H. genesti Giachino \& Vailati, 2005 del "gruppo di H. stefani", entrambe del M. Pasubio, è una delle specie di Halbherria che raggiungono le massime quote finora accertate. I pochi esemplari raccolti sono stati catturati, con l'uso di trappole, in due delle innumerevoli caverne militari, scavate durante la Grande Guerra, sparse nei dintorni del rifugio Damiano Chiesa, ubicate una a quota 1950 $\mathrm{m}$ e l'altra, poco distante, a quota $1860 \mathrm{~m}$. È da rilevare poi che in queste stazioni, $H$. carlini $\mathrm{n}$. sp. convive con Hartigiella baldensis Müller, 1928, presente con un effettivo evidentemente più consistente (Carlin, com. pers.). Gli scarsi esemplari di Halbherria, infatti, raccolti già nel 2013, non erano stati subito riconosciuti e isolati in quanto frammisti a quelli ben più numerosi di Hartigiella baldensis. Ricordo che quest'ultima specie era stata segnalata sul M. Altissimo già quarant'anni or sono (Vailati, 1976; 1977) di un'altra caverna militare presso Malga Campo (A. Martinelli legit) che, sebbene ubicata ad una quota un po' meno elevata $(1500 \mathrm{~m})$, si trova anch'essa nella stessa zona del M. Altissimo, molto prossima alle stazioni della nuova specie di $\mathrm{Hal}$ bherria. Oltre a Hartigiella baldensis, tra la fauna associata (A. Carlin legit, com. pers.), sono da segnalare Duvalius baldensis baldensis (Putzeys, 1870), Choleva glauca Britten, 1918, Catops nigricans (Spence, 1815), Omalium validum Kraatz, 1857, Pella humeralis (Gravenhorst, 1802), Atheta fungi (Gravenhorst, 1806).

\section{CONSIDERAZIONI BIOGEOGRAFICHE}

La scoperta di questa nuova specie di Halbherria risulta di un certo interesse dal punto di vista zoogeografico, in quanto ripropone un tema già più volte 
affrontato e discusso in numerose occasioni e da parte di diversi Autori, costituendo un nuovo elemento utile ad approfondirne l'analisi. Il ruolo e il significato del massiccio del Baldo nella distribuzione nota di determinati endemiti prealpini, nonché il ruolo della stessa valle dell'Adige in quanto "barriera" sono stati trattati, anche se a volte in modo marginale, in relazione all'analisi di taxa, di livelli specifici, generici e sopragenerici, appartenenti a svariati gruppi sistematici.

Sono ben noti i numerosi casi di taxa - specifici e/o generici - che, sovrapponendo i loro areali sul Monte Baldo, li estendono ora a occidente di esso, nelle Prealpi Bresciane, ora a oriente dello stesso, sui rilievi a est della valle dell'Adige, facendo assumere a tale massiccio un ruolo di interposta "cerniera" (Ruffo, 1938, 1950; Vailati, 1976, 1988, 1993a; Chemini \& Tamanini, 1981; Minelli \& Ruffo, 1989; Caoduro et al., 1994; Casale \& Vigna Taglianti, 2005; Giachino \& Vailati, 2005). Non è il caso di ripeterne in questa sede i tanti esempi, e nemmeno di soffermarsi sui più volte citati casi di areali che sono estesi sia a est che a ovest, come avviene per i generi Trogloiulus (Diplopodi), Balkanoroncus (Pseudoscorpioni), Lessinodytes (Coleotteri Carabidi), Rhaetiella (Coleoptera Cholevidae) o, ancora, come avviene per la "serie filetica di Boldoria", presente a est dell'Adige con i generi Monguzziella e Miettiella (Vailati, 1993a; Piva, 2016), nel genere Leptusa e in alcuni Leptotyphlinae (Coleoptera Staphylinidae) (Pace, 1978a, 1978b), nonché nel genere Baldorhynchus (Coleoptera Curculionidae), ampiamente trattato nel recente lavoro di Bellò et al. (2016). Per ciò che può interessare il caso della nuova specie di Halbherria, è opportuno sottolineare che, in tempi recenti, si susseguono sempre più spesso le scoperte di taxa che - appartenenti a linee tipicamente diffuse nelle Prealpi Venete (Sbordoni et al., 1982; Zoia, 1998; Vailati, 1993; Latella \& Sbordoni, 2002; Giachino \& Vailati, 2005), i cui areali si sarebbero teoricamente dovuti arrestare a est della valle dell'Adige - si rivelano presenti anche sul Baldo, rimettendo ogni volta in discussione il ruolo di "barriera" di tale valle e le modalità con le quali certi elementi l'abbiano in qualche modo superata. I riferimenti più prossimi al nostro caso, in parte già altrove discussi (Giachino \& Vailati, 2005; Vailati, 2017), riguardano in particolare due generi di Coleotteri: Orotrechus (Carabidae) e Aphaotus (Cholevidae). Il primo genere, diffuso nelle Alpi orientali dai Lessini fino alla Carinzia e alla Stiria, a sud fino alla Croazia
(Vigna Taglianti, 1982) è presente sul Baldo con Orotrechus vicentinus martinellii Daffner, 1987 (Casale \& Vigna Taglianti, 2005), mentre il secondo, endemico delle Prealpi Venete, ha rivelato la propria presenza sul Baldo più recentemente con Aphaotus martinellii Giachino \& Vailati, 2005. Ora, è pleonastico dire "inaspettatamente", fa la propria comparsa anche il genere Halbherria, finora noto esclusivamente su alcuni rilievi compresi tra l'altopiano dei Sette Comuni e i Lessini veronesi. Come Aphaotus, anche Halbherria appartiene a quel contingente di Cholevidae della "serie filetica di Aphaotus" che, con altre linee di Leptodirini "teleomorfi" (Jeannel, 1924; Sbordoni et al., 1982; Giachino et al., 1998), quali i generi delle "serie filetiche di Neobathyscia e di Aphaobius", sono diffusi principalmente nella fascia prealpina orientale e dinarica, delimitata a ovest appunto dalla Valle dell'Adige.

In particolare, la distribuzione del genere $\mathrm{Hal}$ bherria (Fig. 17) vede concentrarsi le specie appartenenti al "gruppo di H. tamaninii" fra l'altopiano di Folgaria (H. tamaninii), i massicci del Pasubio $(H$. pasubiana) e del Carega (H. pacei) e gli alti Lessini (H. pivai e $H$. vericoi), oltre che sul massiccio del Baldo con $H$. carlini n. sp.; le specie del "gruppo di H. stefani" sono distribuite tra gli alti Lessini (H. zorzii), il Pasubio (H. genesti) e, con una specie (H. stefani) ad areale più ampio, sul Carega, sull'Altopiano di Folgaria e su quello dei Sette Comuni; su quest'ultimo altopiano troviamo infine l'unica specie del monotipico "gruppo di H. cimbrica" (Giachino \& Vailati, 2005), della quale è stato ampliato l'areale con il ritrovamento anche alla Grotta di Costalta (Carlin, 2007).

Ora, se osserviamo non solo la distribuzione di tutte le specie note di Halbherria, ma anche di quelle di Aphaotus - dal momento che i due generi sovrappongono in buona parte i propri areali sui medesimi rilievi, anche con alcune specie simpatriche - non possiamo non notare una certa coincidenza topografica delle stazioni con i margini di aree glacializzate durante l'Ultimo Massimo Glaciale (UMG); la posizione della maggior parte di esse ci appare come "arroccata" su singoli massicci che, per la loro altitudine, sono stati risparmiati dai ghiacciai vallivi, che li hanno lambiti e spesso in parte accerchiati (Fig. 18). Questa rappresentazione ha deposto a favore, da lungo tempo e in una vasta letteratura, dell'idea affascinante dei "massicci di rifugio" in alta quota, nei quali sarebbero 


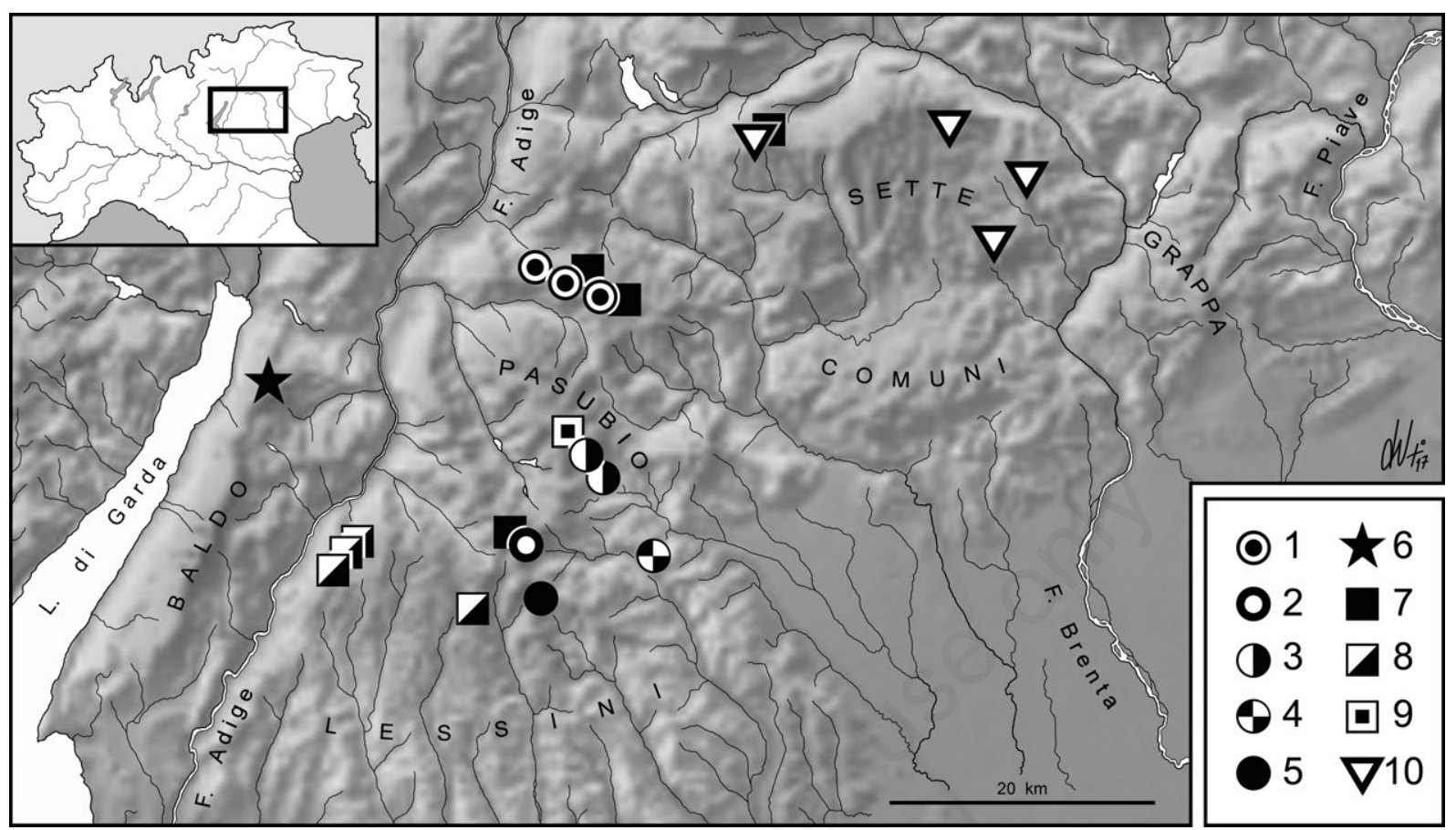

Fig. 17. Distribuzione delle specie note del genere Halbherria. 1) H. tamaninii; 2) H. pacei; 3) H. pasubiana; 4) H. pivai; 5) H. vericoi; 6) H. carlini n. sp.; 7) H. stefani; 8) H. zorzii; 9) H. genesti; 10) H. cimbrica.

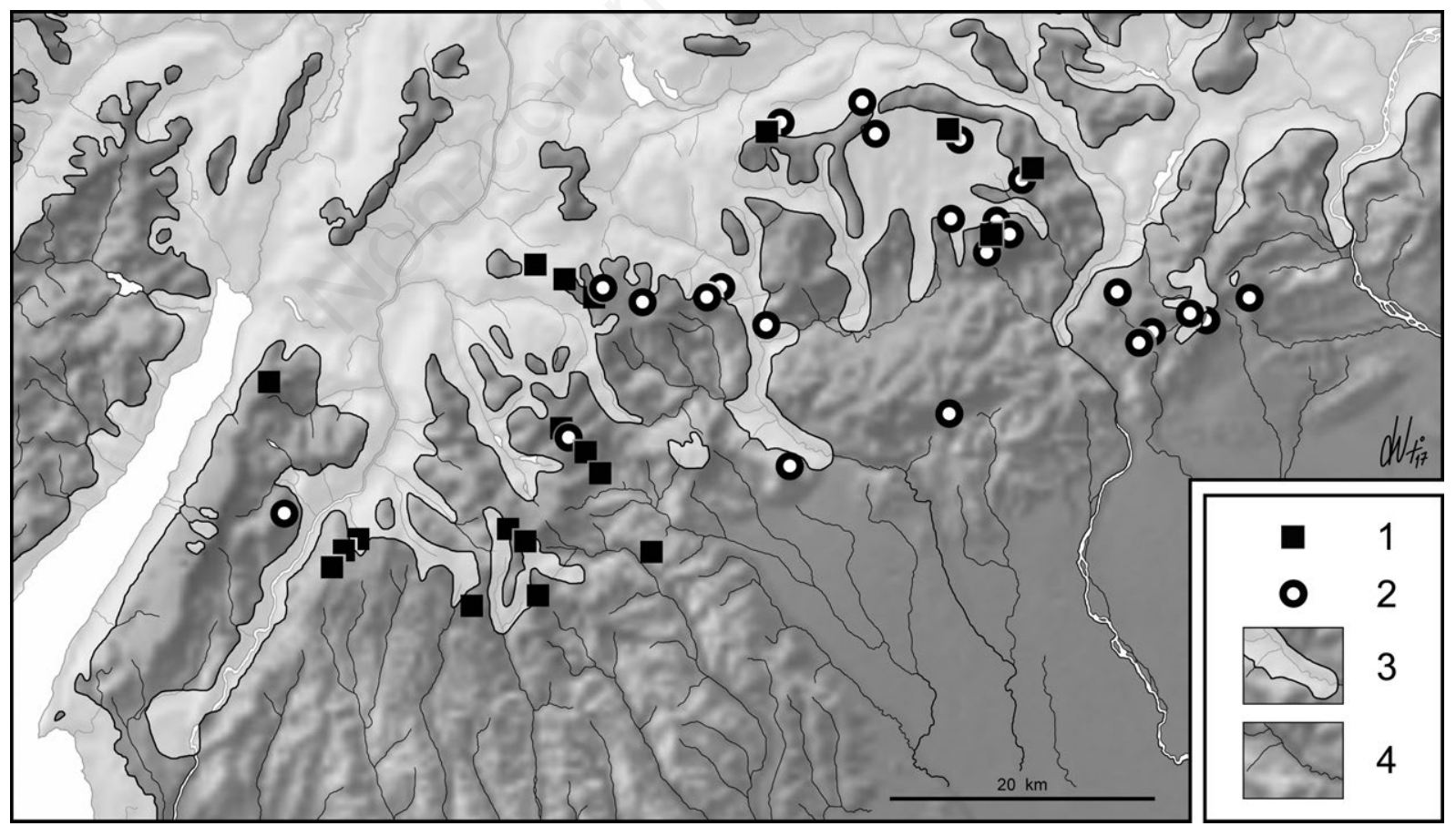

Fig. 18. Distribuzione delle stazioni note dei generi Halbherria e Aphaotus in rapporto all'estensione dell'Ultimo Massimo Glaciale. 1) Halbherria; 2) Aphaotus; 3) ghiacciai; 4) territori liberi dai ghiacci. 
sopravvissute le popolazioni sfuggite alla devastazione glaciale, che si sarebbero quindi differenziate in specie diverse in virtù di un forzato isolamento.

In più di un'occasione ho avuto modo di ribadire che personalmente non concordo con tale visione (Vailati, 1988, 1991; anche in Giachino \& Vailati, 2005). Argomentazioni già altrove riferite mi hanno sempre fatto propendere per un approccio diverso al problema e non sarebbe il caso di riprenderle per l'ennesima volta, se non fosse per sottolineare nuovamente che il frequente utilizzo che viene fatto del fattore "glacialismo alpino", per argomentare fatti biogeografici, è troppo spesso trattato con estrema e ingannevole semplificazione. Fenomeni di vicarianza per isolamento, probabili responsabili di processi di differenziazione, vengono sempre associati al quadro offerto dall'Ultimo Massimo Glaciale, come se tale situazione fosse generalizzabile, esclusiva, in pratica riassuntiva di un'unicità dell'intero Pleistocene, come, al contrario, non è stata. Oggi sappiamo dell'estrema complessità di un'epoca che è caratterizzata dall'alternanza di molteplici cicli glaciali/interglaciali, più di una trentina, molti dei quali ben più antichi dell'UMG, e resa ancora più complessa dalle innumerevoli pulsazioni stadiali/interstadiali, con oscillazioni climatiche tali da aver giocato un ruolo determinante nel favorire più episodi di rimaneggiamento di precedenti distribuzioni, con possibili contrazioni ed espansioni $\mathrm{e}$, soprattutto, con la risalita in più fasi dei rilievi montuosi locali, intesa come risposta ipsofila indotta però dalle fasi interglaciali che, per elementi microtermofili, può meglio spiegare l'isolamento e i conseguenti episodi di differenziazione, come ho più volte precisato (Vailati, 1988, 1990, 1991, 1993b) e come parrebbero concordare anche le opinioni in campo fitogeografico (Ravazzi, 1997). Se oggi la distribuzione delle stazioni note ci appare ai margini dei ghiacciai dell'UMG, non è detto quindi che siano sempre state, ripetutamente, in tali posizioni. Dove potevano essere durante un ciclo precedente? E prima ancora? Queste domande, ovviamente, non hanno mai una risposta precisa e illuminante.

D'altra parte, è assodato che rimaneggiamenti di tale portata - implicitamente quindi con migrazioni eterocrone di popolazioni e loro altrettanto eterocrona allopatria - possono essersi realizzati anche in tempi abbastanza recenti, come appunto durante gli ultimi cicli fino al Tardoglaciale. A differenza degli episodi glaciali più antichi, i cui dettagli sono sempre più sfu- mati man mano che si va indietro nel tempo, gli stadi più recenti sono molto ben documentati e ci parlano tra l'altro anche di numerose riprese del glacialismo, ancora nel Tardoglaciale, con diverse avanzate anche durante il generale ritiro delle grandi lingue vallive, con alternanze climatiche e vegetazionali molto variabili in rapporto alle diverse situazioni a scala locale (Baroni et al., 2001; Orombelli et al., 2004, 2005; Ravazzi, 2002, 2003, 2005; Ravazzi et al., 2004, 2007); è difficile tuttavia ricondurre la maggior parte dei fenomeni di differenziazione alla più recente espansione glaciale. Oltretutto, se consideriamo l'UMG dal punto di vista temporale, dobbiamo convenire che tale fase costituisce un fatto molto recente, più facilmente responsabile degli ultimi rimaneggiamenti di areali, ma più difficilmente di episodi di differenziazione per vicarianza che, verosimilmente, dovrebbero avere a disposizione un arco di tempo ben maggiore e pressioni selettive indotte dal ripetersi ciclico di un grande numero di alternanze bioclimatiche. Casi non rari di popolazioni allopatiche assolutamente indifferenziate deporrebbero infatti a favore di disgiunzioni, anche notevoli, affatto recenti, verosimilmente legate all'ultima fase di deglaciazione tardo-pleistocenica, come nel caso delle popolazioni indifferenziate di Halbherria stefani (Breit, 1914) presenti sia a nord sulla Cima Manderiolo, sia sull'altopiano di Folgaria, sia ancora nel gruppo del Carega (Giachino \& Vailati, 2005) o di quelle numerose di Monguzziella grottoloi Vailati, 1993 presente anch'essa sull'altopiano di Folgaria, nel gruppo Pasubio-Carega oltre che negli alti Lessini (Piva, 2016) o, ancora e altrove, del caso emblematico della stazione di Stoppaniola robiati (Reitter, 1889) insediata nei conglomerati di Paderno d'Adda, a sud dell'anfiteatro morenico lariano (Vailati, 1991).

E proprio in virtù della eterogeneneità dell'avvicendamento dei numerosi episodi di transizione succedutisi in un arco di tempo plio-pleistocenico, non credo che tutto questo periodo si possa semplificare con la statica immagine dell'UMG e dipingere tout court il Monte Baldo come "un'isola tra i ghiacci". La complessità delle variazioni climatiche, avvenute durante il Pleistocene, ci offre un quadro nel quale ci risulta sempre più difficile pensare che il Baldo sia rimasto poi così isolato dai Lessini o, se lo è stato, non lo è stato sempre e in modo continuativo. Se in determinati momenti il massiccio è stato circondato e quasi "chiuso" dalle discese vallive dei ghiacciai del Garda e dell'Adige, penso che non si possa enfatizzare tale 
situazione di isolamento come esclusiva e determinante, poiché in altre occasioni di transizione ha certamente offerto a molti elementi provenienti da est la possibilità di essere colonizzato.

Anche per Halbherria, quindi - in cui, accanto alla più ampia distribuzione sui rilievi delle Prealpi Venete Occidentali, si affaccia ora una specie che ha inequivocabilmente raggiunto da est il massiccio del Baldo - si potrebbe ipotizzare un modello di diffusione analogo a quello illustrato da Giachino \& Vailati (2005: p. 252-254) per il genere Aphaotus, schematico ed esemplificativo in quel caso, ma probabilmente estensibile anche ad altri generi diffusi nella medesima area prealpina.

Tuttavia, dobbiamo ammettere che tali scenari mantengono comunque il puro valore di ipotesi di lavoro, poiché non è mai facile trovare le precise correlazioni, soprattutto in termini temporali, fra $\mathrm{i}$ casi di volta in volta in esame e quindi non è nemmeno troppo lecito che vengano facilmente generalizzate a situazioni geografiche e/o a gruppi sistematici fra loro diversi. Alla luce di un'attenta analisi degli eventi paleogeografici e paleoclimatici locali, basata sulle conoscenze geologiche e glaciologiche dell'area in questione, non escludo di potere ipotizzare per il massiccio del Baldo episodi di colonizzazione da est anche anteriori alle principali glaciazioni pleistoceniche, come del resto già ipotizzato per altri taxa e per altri settori geografici (Giachino, 1993; Latella \& Sauro, 2007; Latella et al., 2012; Vailati, 2017), verosimilmente potuti avvenire già nel Pliocene, se non ancora prima, forse in questo caso particolare favoriti anche da una topografia alquanto differente dall'attuale.

\section{Sulla “BARrierA” Biogeografica della Valle DELL'AdigE}

La geografia dell'area in esame mostra motivi di particolare interesse, se rapportata agli eventi tardo terziari e quaternari che l'hanno interessata, modellandola dal punto di vista geologico, geomorfologico, glaciologico, idrologico e, conseguentemente, climatico e biologico.

Anche se un eccessivo approfondimento esula dal principale scopo di questa nota, vale la pena di ricordare brevemente che l'evoluzione avvenuta nell'assetto geo-idrografico, già prima degli eventi glaciali pleistocenici, ha fortemente condizionato la topografia della media e bassa Valle dell'Adige (Val Lagarina) e, indirettamente e per ciò che ci riguarda, rende in qual- che modo più aleatoria l'idea di un totale isolamento del Baldo dai Lessini.

Pur essendo quasi tutta la Valle dell'Adige impostata su un grande disturbo tettonico con andamento NNE-SSW, geneticamente correlato e quindi parallelo alla "linea delle Giudicarie" (AA.VV. in Castellarin \& Vai, 1982; Castellarin et. al., 1992), la complessità dell'evoluzione tettonica locale determina fin dal Miocene un sistema idrografico organizzato in modo molto diverso dall'attuale, che viene ereditato nelle grandi linee fino verso la fine del Pliocene e l'inizio del Pleistocene. L'Adige non transita la valle interamente verso sud fino a sfociare in pianura, come avviene attualmente, ma è costretto a percorsi alternativi dalla presenza di sbarramenti orografici trasversali alla valle stessa: la propaggine di Candriai che scende dal Bondone (destra idrografica) è unita al M. Calisio (sinistra idrografica), determinando uno sbarramento della valle appena a nord di Trento. Appena a sud della città, un'altra digitazione dello stesso gruppo del Bondone è unita al M. Marzola, creando un ulteriore sbarramento. Tale situazione obbliga l'Adige, che ha da poco ricevuto il Noce e l'Avisio e sta proseguendo verso SSW, a deviare - o sarebbe meglio dire ad andare diritto in tale direzione, che è quella dei principali sistemi tettonici - attraverso la valle di Terlago-Vezzano-Sarche (Valle dei Laghi) dove si unisce al basso Sarca per confluire nel bacino del Garda (Trevisan, 1942; Corrà, 1973, 1974; Sauro, 2005). Più a sud, nel tratto tra gli abitati di Ala e di Avio, un ulteriore sbarramento orografico collega in pratica il M. Castelberto (area lessinica) al M. Vignola (area baldense).

Tali sbarramenti determinano di fatto tre bacini idrografici indipendenti (Corrà, 1974): i) a nord quello "atesino" che come detto, attraverso la Valle dei Laghi, scorre verso il Garda; ii) nel mezzo quello "roveretano" che, raccolte le acque del Fersina e della Valle di Piné da nord (che aggirano a sud il M. Marzola, scendendo attraverso la valle di Vigolo Vattaro) e della Vallarsa e Valle dei Ronchi da sud, defluisce pure nel Garda, ma attraverso la sella di Loppio-Nago; iii) a valle dello sbarramento Castelberto-Vignola, il più modesto bacino "baldo-lessineo", nel quale scorre un breve fiume verso sud fino all'altezza di Rivoli, ma ancora senza raggiungere la pianura, costretto anch'esso a versare le proprie acque nel golfo di Garda dall'ulteriore sbarramento della Chiusa di Ceraino, propaggine orografica del M. Pastello (Fig. 19). 
Questo è verosimilmente lo scenario fino alla fine del Pliocene, nel quale si muoveranno le prime invasioni glaciali pleistoceniche, accompagnate dall'evolversi di tale assetto idrografico, da esse stesse in parte favorito. Ora, è necessario focalizzare l'attenzione su quello che Corrà (1974) chiama bacino "baldo-lessineo". Tale breve bacino a sud di Avio - dove colpisce il fatto che anche attualmente la valle sia più stretta della porzione atesina che le sta più a nord - raccoglie le

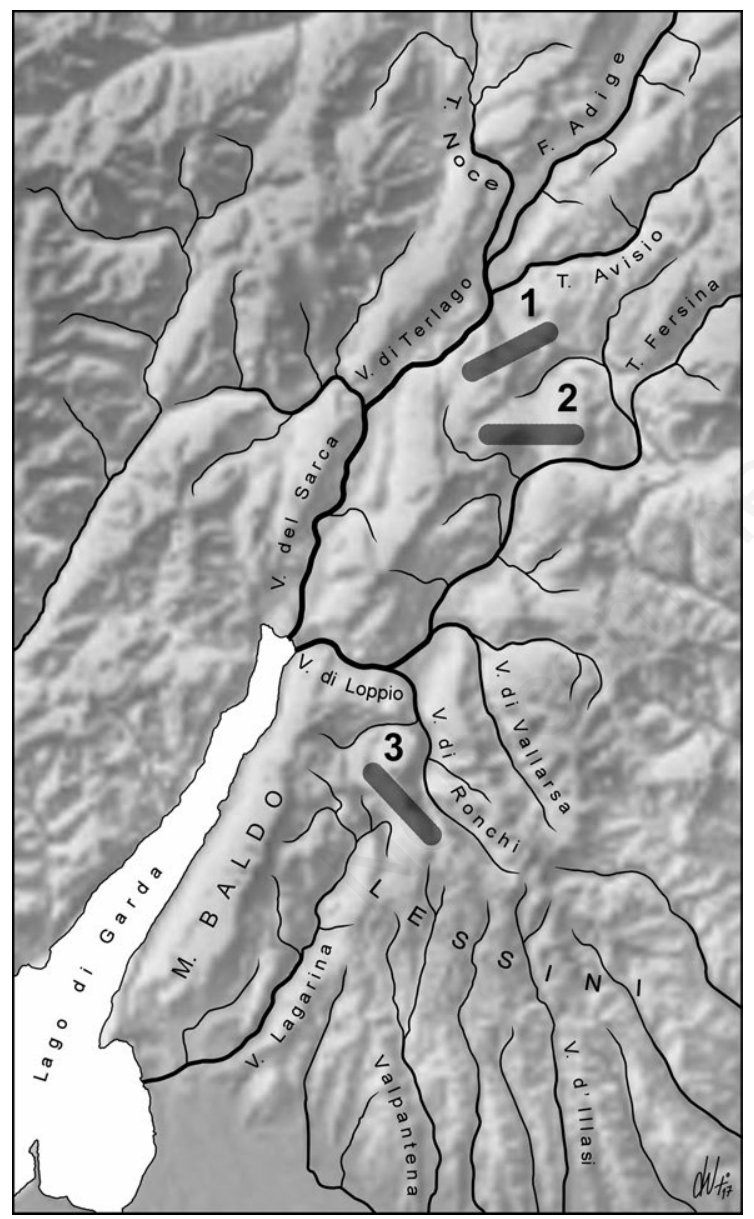

Fig. 19. Rappresentazione del possibile scenario oro-idrografico della media e bassa Valle dell'Adige alla fine del Pliocene, in cui si evidenziano, a seguito degli sbarramenti vallivi, le tre distinte reti idrografiche immissarie del bacino del Garda. 1) sbarramento Calisio-Candriai; 2) id. MarzolaBondone; 3) id. Castelberto-Vignola e possibile collegamento Baldo-Lessini (ricostruzione ridisegnata e adattata da Corrà, 1974). acque del versante occidentale lessineo fino al Corno d'Aquilio e del versante orientale del Baldo; oltretutto, al momento la valle non è stata ancora approfondita dall'erosione e dall'esarazione glaciale e appare non molto più lunga delle attuali Valpantena, Val di Squaranto o della Valle d'Illasi, tanto che, in un certo senso, può essere considerata come "la più occidentale delle valli lessinee" (Corrà, 1973, 1974), anche senza voler invocare situazioni più antiche, pre-plioceniche, in cui si possa

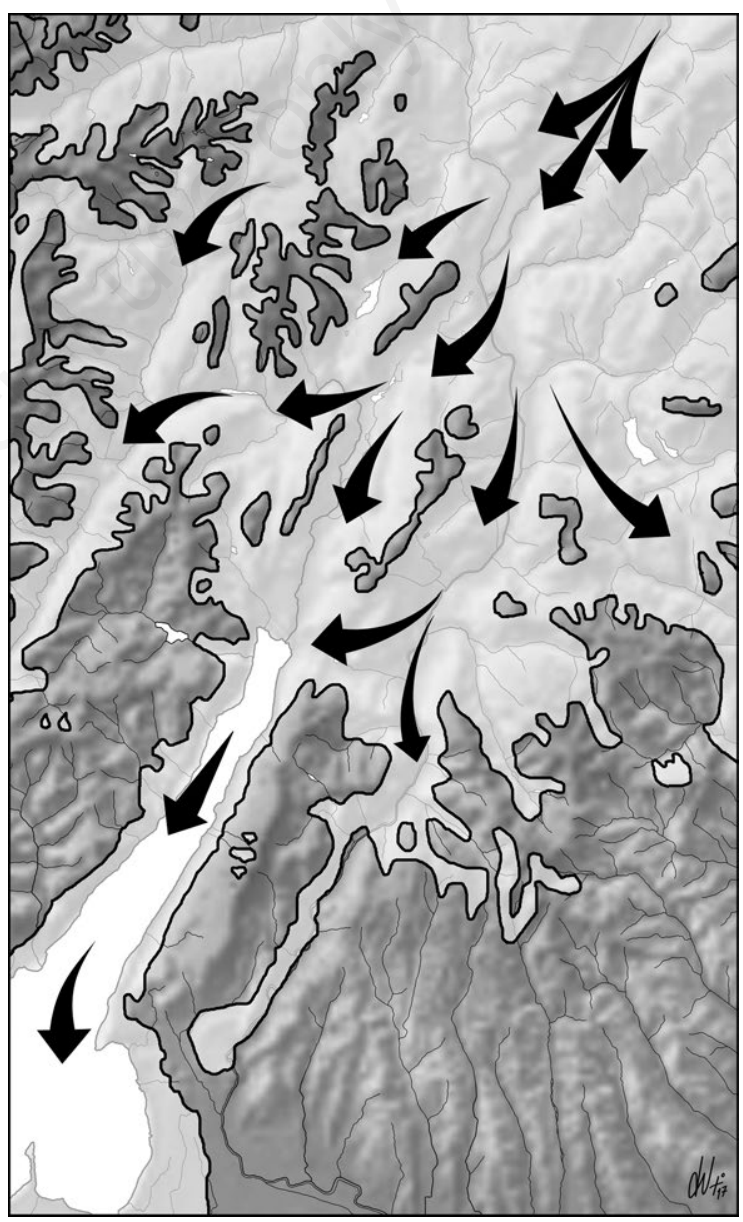

Fig. 20. Rappresentazione semplificata dell'Ultimo Massimo Glaciale che ha interessato la media e bassa Valle dell'Adige, in cui di evidenzia la modesta lingua glaciale avanzata nella bassa Valle Lagarina, rispetto alle poderose transfluenze avvenute più a monte, e in cui il massiccio del M. Baldo appare "circondato" dai ghiacciai di detta valle e del Garda. (fonti: Antonioli \& Vai, 2004; Orombelli et al., 2004). 
immaginare una breve "valle locale, incisa nell'ambito dell'esteso altopiano M. Baldo-Lessini" (Sauro, 2005). Solo nel Pleistocene superiore - per "cattura fluviale" dovuta all'azione combinata dell'erosione regressiva della valle da sud e dell'azione del ghiacciaio atesino da nord, unita ai processi periglaciali di gelifrazione, che contribuiscono allo smantellamento dello sbarramento Castelberto-Vignola - l'Adige può finalmente scorrere nella bassa Val Lagarina, sia pure ancora fino alla Chiusa di Ceraino, aperta e superata solo nell'ultima fase glaciale.

Anche il succedersi degli episodi glaciali del Pleistocene ci offre un quadro su cui riflettere. Il grande ghiacciaio dell'Adige, dove il termine "grande" vuole riferirsi all'estesa zona di accumulo presente a monte nelle Alpi Retiche, nelle sue discese è stato più volte costretto, circa poco prima della città di Trento, a diverse transfluenze (Fig. 20), che hanno prodotto lingue vallive di dimensione più importante sia verso la valle benacense, dilagando in pianura con l'ampio lobo dell'anfiteatro del Garda, sia ancora più a ovest, scendendo attraverso la conca di Stenico nelle Giudicarie fino a raggiungere il lago d'Idro, mentre a est altre lingue vallive, sempre transfluenti dal bacino dell'Adige, scendevano nelle valli d'Astico e del Brenta, circondando i massicci del Carega-Pasubio e dell'altopiano dei Sette Comuni, isolando quest'ultimo dal Monte Grappa. Una lingua ben più modesta invece, dovuta al controllo morfologico, è scesa nella Val Lagarina, tanto da arrestarsi facilmente pur'essa all'altezza di Rivoli, alla Chiusa di Ceraino (Sauro, 2005).

Anche se i dati recenti sulla posizione degli apparati morenici mostrano quote inferiori di oltre 100 $\mathrm{m}$ della copertura glaciale rispetto a quanto sostenuto in passato (Penk \& Brückner, 1909) e come riportato nelle suggestive carte di Castiglioni (1940) e di Habbe (1961), depositi glaciali precedenti l'ultima glaciazione sono riconosciuti a vari livelli e quote comunque più elevate di quelle raggiunte durante l'Ultimo Massimo Glaciale nel sistema del Garda, attraverso la valle dei Laghi, a testimonianza delle ripetute transfluenze avvenute attraverso tale valle durante tutto il Pleistocene (Bassetti \& Borsato, 2007; Borsato, 2010). In mezzo alle transfluenze sopra ricordate, solo alcuni massicci, come ad esempio la Paganella, il Bondone, il Becco di Filadonna, emergevano come altrettanti piccoli nunatak.

\section{CONCLUSIONI}

La scoperta di questa nuova specie assume un particolare e importante valore in quanto, oltre ad ampliare le nostre conoscenze sul genere Halbherria, ci consente di ripercorrere un tema che, anche se più volte trattato, allo stesso tempo è sempre stato caratterizzato da dubbi e incertezze: il significato, in termini di validità, sia in senso spaziale che temporale, del concetto di "barriera" della Valle dell'Adige, intesa come limite occidentale nei confronti di determinati gruppi zoologici che, in base alle conoscenze di un passato non tanto remoto, estendevano i loro areali a oriente di essa, senza superarla. Le scoperte, avvenute negli ultimi decenni, di alcuni elementi di tali gruppi, legati all'ambiente sotterraneo, cui si aggiunge la nuova specie qui descritta, pone in risalto la loro presenza anche nel massiccio del Monte Baldo e, di concerto, anche la necessità di una possibile risposta ai numerosi quesiti che tale situazione richiede.

Sulla base dell'analisi della letteratura riguardante la paleoidrografia e il glacialismo dell'area considerata, non è da escludere l'ipotesi che penetrazioni da est verso ovest, di elementi diffusi sulle Prealpi Venete, si siano verificate verosimilmente ancora sul finire del Pliocene superiore, in ogni caso anteriormente alle più antiche fasi glaciali del Pleistocene, in un tempo durante il quale l'assetto della rete idrografica, e quindi il sistema vallivo, della media e bassa Valle dell'Adige - ereditati dall'evoluzione tettonica in atto fin dal Miocene - erano tali da consentire un possibile collegamento tra gli alti Lessini e la porzione settentrionale del massiccio del M. Baldo.

\section{RINGRAZIAMENTI}

Sono grato innanzitutto all'amico Andrea Carlin, che ha voluto affidarmi in studio il frutto della sua interessante scoperta, con la quale ci fornisce un'ulteriore occasione di approfondimento delle conoscenze della componente endemica di quella straordinaria biodiversità presente con grande dovizia sulle nostre Prealpi. Ringrazio inoltre gli amici Achille Casale, Pier Mauro Giachino e Massimo Meregalli per la consueta pazienza con cui hanno riletto il mio manoscritto e per le loro utili osservazioni. 
BIBLIOGRAFIA

Antonioli F., VAi G.B. (eds.), 2004 - Litho-palaeoenvironmental maps of Italy during the Last Two Climatic Extremes. Map 1, Last Glacial Maximum. 32nd International Geological Congress, Firenze.

Baroni C., Bruschi G., Veronese L., Zanchetta G., 2001 - Younger Dryas to early Holocene palaeoenvironmental evolution of the Lake Terlago (Southern Alps). Geografia Fisica e Dinamica Quaternaria, 24: 13-24.

Bassetti M., Borsato A., 2007 - Evoluzione geomorfolocica della bassa Valle dell'Adige dall'ultimo massimo glaciale: sintesi delle conoscenze e riferimenti ad aree limitrofe. Studi Trentini di Scienze Naturali, Acta Geologica, 82: 29-40.

Bellò C., Osella G., Baviera C., 2016 - A taxonomic revision of the genus Baldorhynchus (Di Marco e Osella, 2002) stat. n. (Coleoptera, Curculionidae, Entiminae). Zootaxa, 4070(1): 101.

Borsato A., 2010 - V. Quaternario. In: Avanzini M, Bargossi G.M., Borsato, A., Selli L. (eds.), Note illustrative della Carta Geologica d'Italia alla scala 1:50.000. Foglio 060 Trento. ISPRA, Servizio Geologico d'Italia, pp. 117-176.

Cadoduro G., Osella G., Ruffo S., 1994 - La fauna cavernicola della Regione Veronese. Memorie del Museo Civico di Storia Naturale, Verona, $2^{\circ}$ s., 11: 144 pp.

Carlin A., 2007 - Tre troglobi, ma non solo..., caccia grossa sotto terra. La coleotterofauna cavernicola della Grotta di Costalta (n. VT/TN 14); alcune inedite segnalazioni entomologiche per il Trentino. Natura Alpina, 58(3/4): 69-78.

Casale A., Vigna Taglianti A., 2005 - Coleotteri Caraboidei delle Alpi e Prealpi centrali e orientali, e loro significato biogeografico. XXXV Congresso della Società Italiana di Biogeografia, Rabbi, 2004. Biogeographia, 26: 129-201.

Castellarin A., Cantelli L., Fesce A.M., Mercier J., Picotti V., Pini G.A., Prosser G., Selli L., 1992 - Alpine compressional tectonics in the Southern Alps. Relationships with the N-Apennines. Annales Tectonicae, 6(1): 62-94.

Castellarin A., VAi G.B. (eds.), 1982 - Guida alla geologia del Sudalpino centro-orientale. Guide Geologiche Regionali, Società Geologica Italiana, 24: 381 pp.

Castiglioni B., 1940 - L'Italia nell'Era Quaternaria. Carta delle Alpi nel Glaciale. Tav. 3 in: Dainelli G. (ed.), Atlante fisicoeconomico d'Italia, Milano.

Chemini C., Tamanini L., 1981 - Considerazioni sulla fauna del Monte Baldo. In: AA. Vv., Il Monte Baldo nei suoi aspetti naturalistici e antropici. Natura alpina, 32(27): 77-82.

Conci C., TAMAnini L., 1951 - Revisione del genere «Aphaotus» Breit e descrizione di un nuovo genere di Coleotteri troglobi. Studi Trentini di Scienze Naturali, 28: 111-144.

CORRÀ G., 1973 - L'evoluzione morfologica della Valle Lagarina. Natura Alpina, 24(1): 3-19.

CORRÀ G., 1974 - Il ruolo delle glaciazioni quaternarie nelle vicende della Idrografia atesina. Natura Alpina, 25(4): 177-193.

GiACHINo P.M., 1993 - La distribuzione dei generi Binaghites e Bathysciola nelle Alpi Occidentali (Coleoptera Carabidae e Cholevidae). Biogeographia, Lavori della Società Italiana di Biogeografia, (n.s.) 16: 401-424.

Giachino P.M., Vailati D., 2005 - I Cholevidae delle Alpi e Prealpi italiane: inventario, analisi faunistica e origine del popolamento nel settore compreso fra i corsi dei fiumi Ticino e Tagliamento (Coleoptera). XXXV Congresso della Società Italiana di Biogeografia, Rabbi, 2004. Biogeographia, 26: 229-378.

Giachino P.M., Vailati D., Casale A., 1998 - Major questions in the phylogeny and biogeography of Cholevidae (Coleoptera), with emphasis on the subfamily Leptodirinae, pp. 179-209. In: GIACHINO P.M. \& PECK S.B. (eds.), Phylogeny and Evolution of Subterranean and Endogean Cholevidae (=Leiodidae Cholevinae). Proceedings of a Symposium (30 August 1996). XX International Congress of Entomology, Florence (Italy), 1996. Atti del Museo Regionale di Scienze Naturali, Torino, $296 \mathrm{pp}$.

HabBe K.A., 1961 - Zur Klimatischen Morfologie der Alpensudolrands-Unterschungen in den Morenaanphitheatern der Etsch und Gardasee. Nachr. Acad. Wiss. Gottingen, 10: 179-203.

JeAnNel R., 1924 - Monographie des Bathysciinae. Archives de Zoologie Expérimentale et Générale, 63: 436 pp.

Latella L., Sauro U., 2007 - Aspects of the evolution of an important geo-ecosystem in the Lessinian Mountain (Venetian Prealps, Italy). Acta Carsologica, 36: 69-75.

Latella L., Sbordoni V., 2002 - Fauna delle grotte: 339-358. In: Minelli A., Chemini C., Argano R., Ruffo S. (eds), La fauna in Italia. Touring Club Italiano e Ministero dell'Ambiente e della Tutela del Territorio, 448 pp.

Latella L., Verdari N., GobBi M., 2012 - Distribution of terrestrial cave-dwelling Arthropods in two adjacent Prealpine Italian areas with different glacial histories. Zoological Studies, 51(7): 1113-1121.

Minelli A., Ruffo S., 1989 - La fauna cavernicola veneta. In: Mietto P. \& Sauro U. (eds.), Le grotte del Veneto. Regione del Veneto e La Grafica Editrice, Verona: 49-60.

Orombelli G., RavazZI C., CitA M.B., 2005 - Osservazioni sul significato dei termini LGM (UMG), Tardoglaciale e postglaciale in ambito globale, italiano e alpino. Il Quaternario, Italian Journal of Quaternary, 18(2): 147-155.

Orombelli G., Tanzi G., Ravazzi C., 2004 - Glacier extent over the Italian Alps during the LGM. In: Antonioli F., Vai G.B. 
(eds.), Litho-palaeoenvironmental maps of Italy during the Last Two Climatic Extremes. Explanatory Notes. $32^{\text {nd }}$ International Geological Congress, Firenze: 23-24.

Pace R., 1978a - Le specie di Leptusa Kr. di Cima Carega (Trentino) (Coleoptera, Staphylinidae). Studi Trentini di Scienze Naturali, Acta Biologica, 55: 157-170.

PACE R., 1978b - Revisione di alcune forme di Leptotyphlinae del Trentino, del Veneto e del Friuli (Coleoptera, Staphylinidae). Studi Trentini di Scienze Naturali, Acta Biologica, 55: 193-205.

Penck A., BrÜcKner E., 1909 - Die Alpen im Eiszeitalter. III. Die Eiszeiten in den Sudalpen und im Bereich der Ostabdachung der Alpen. Leipzig, 1200 pp.

PIVA E., 1984 - Nuovi Bathysciinae raccolti in cavità del Vicentino (Coleoptera Catopidae Bathysciinae). Giornale Italiano di Entomologia, 2: 203-214.

PIVA E., 2016 - Miettiella vespertilio nuovo genere nuova specie dei Monti Lessini Veronesi (Veneto, Italia) (Coleoptera Cholevidae Leptodirinae). Bollettino della Società entomologica italiana, 148(2): 63-70.

RaVAzZI C., 1997 - Inquadramento fitogeografico delle Prealpi Lombarde e della Regione Insubrica: problemi geoecologici e paleobotanici. Quaderni del Parco Monte Barro, 4: 63-79.

RAVAZZI C., 2002 - Late Quaternary history of spruce in southern Europe. Review of Palaeobotany and Palynology, 120: 131-177.

RAVAZZI C., 2003 - An overview of the Quaternary Continental stratigraphic units based on biological and climatic events in Italy. Il Quaternario, Italian Journal of Quaternary Sciences, 16(1bis): 11-18.

RAVAzZI C., 2005 - Il Tardoglaciale: suddivisione stratigrafica, evoluzione sedimentaria e vegetazionale nelle Alpi e in Pianura Padana. Studi Trentini di Scienze Naturali, Acta Geologica, 82: 17-29.

Ravazzi C., Orombelli G., Tanzi G., ClimeX group, 2004 - An outline of the flora and vegetation of Adriatic Basin (Northern Italy and eastern side of the Apennine) during the Last Glacial Maximum. In: Antonioli F., Vai G.B. (eds.), Lithopalaeoenvironmental maps of Italy during the Last Two Climatic Extremes. Explanatory Notes. $32^{\text {nd }}$ International Geological Congress, Firenze: 15-20.

Ravazzi C., Peresani M., Pini R., Vescovi E., 2007 - Il Tardoglaciale nelle Alpi Italiane e in Pianura Padana. Evoluzione stratigrafica, storia della vegetazione e del popolamento antropico. Il Quaternario, Italian Journal of Quaternary Sciences, 20(2): 163-184.

RUFFo S., 1938 - Studio sulla fauna cavernicola della regione veronese. Istituto di Entomologia della Regia Università di Bologna, 10: 70-116.

Ruffo S., 1950 - Descrizione di due nuovi Catopidi cavernicoli del Veronese e osservazioni sul genere Neobathyscia Müll. Memorie del Museo Civico di Storia Naturale, Verona, 2: 125-133.

SAuro U., 2005 - Dal ghiacciaio al fiume. Pp. 23-59. In: Turri E. \& Ruffo S. (eds.), Adige, il fiume, gli uomini, la storia. Cierre ed.,Verona, $396 \mathrm{pp}$.

Sbordoni V., Rampini M., Cobolli Sbordoni M., 1982 - Coleotteri Catopidi cavernicoli italiani. Lavori della Società Italiana di Biogeografia, n. s., VII: 253-335.

TREVISAN L., 1942 - La struttura geologica dei dintorni di Trento. Studi Trentini di Scienze Naturali, 22(3):127-148.

VAILATi D., 1976 - Sulla posizione sistematica di Bathysciola (Hartigiella) baldensis Müller, 1928 con note corologiche, ecologiche e biogeografiche. Natura bresciana, 12: 34-50.

VAILATI D., 1977 - Note corologiche e tassonomiche su alcune specie del genere Boldoria (s.str.) Jeannel. Natura Bresciana, 13: 64-74.

VAilati D., 1988 - Studi sui Bathysciinae delle Prealpi centro-occidentali. Revisione sistematica, ecologia, biogeografia della "serie filetica di Boldoria" (Coleoptera Catopidae). Monografie di Natura bresciana, 11: 332 pp.

VAILATI D., 1990 - Insubriella paradoxa nuovo genere nuova specie di Bathysciinae delle Prealpi italiane. Natura bresciana, 25: 213-229.

Vailati D., 1991 - Nuovi dati sulla distribuzione di Pseudoboldoria robiatii (Reitter, 1889) e considerazioni sulla corologia pleistocenica dei Bathysciinae in Lombardia. Natura bresciana, 26: 223-234.

VAILATI D., 1993a - Monguzziella grottoloi nuovo genere nuova specie delle Prealpi Venete (Coleoptera Cholevidae Leptodirinae). Natura bresciana, 28: 261-278.

VAILATI D., 1993b - Segnalazione di una nuova stazione e revisione morfologica di Cryptobathyscia gavardensis Vailati, 1980 (Coleoptera Cholevidae Leptodirinae). Natura bresciana, 28: 279-285.

VAilati D., 2017 - Revisione tassonomica delle «serie filetiche di Dellabeffaella e di Boldoria» con descrizione di quattro nuovi generi (Coleoptera Cholevidae Leptodirinae). Bollettino della Società Entomologica Italiana, 149 (1): 3-32.

Vigna TAgLianti A., 1982 - Le attuali conoscenze sui Coleotteri Carabidi cavernicoli italiani. Lavori della società Italiana di Biogeografia, n. s., 7: 339-430.

ZOIA S., 1998 - Considerations of the present knowledge of the Italian Cholevidae and their distribution, with particular reference to the hypogean species (Coleoptera), pp. 179-209. In: GiachINo P.M. \& PECK S.B. (eds.), Phylogeny and Evolution of Subterranean and Endogean Cholevidae (=Leiodidae Cholevinae). Proceedings of a Symposium (30 August 1996). XX International Congress of Entomology, Florence (Italy), 1996. Atti del Museo Regionale di Scienze Naturali, Torino, 296 pp. 